University of Nebraska - Lincoln

DigitalCommons@University of Nebraska - Lincoln

$11-16-2012$

\title{
Threshold effects in strong-field ionization: Energy shifts and Rydberg structures
}

\author{
Katarzyna Krajewska \\ University of Warsaw, Poland, kkraj@fuw.edu.pl \\ Ilya I. Fabrikant \\ University of Nebraska-Lincoln, ifabrikant@unl.edu \\ Anthony F. Starace \\ University of Nebraska-Lincoln, astarace1@unl.edu
}

Follow this and additional works at: https://digitalcommons.unl.edu/physicsstarace

Part of the Atomic, Molecular and Optical Physics Commons, Elementary Particles and Fields and String Theory Commons, and the Plasma and Beam Physics Commons

Krajewska, Katarzyna; Fabrikant, llya I.; and Starace, Anthony F., "Threshold effects in strong-field ionization: Energy shifts and Rydberg structures" (2012). Anthony F. Starace Publications. 191. https://digitalcommons.unl.edu/physicsstarace/191

This Article is brought to you for free and open access by the Research Papers in Physics and Astronomy at DigitalCommons@University of Nebraska - Lincoln. It has been accepted for inclusion in Anthony F. Starace Publications by an authorized administrator of DigitalCommons@University of Nebraska - Lincoln. 


\title{
Threshold effects in strong-field ionization: Energy shifts and Rydberg structures
}

\author{
K. Krajewska, ${ }^{1}$ I. I. Fabrikant, ${ }^{2}$ and A. F. Starace ${ }^{2}$ \\ ${ }^{1}$ Institute of Theoretical Physics, Faculty of Physics, University of Warsaw, Hoża 69, 00-681 Warsaw, Poland \\ ${ }^{2}$ Department of Physics and Astronomy, The University of Nebraska, Lincoln, Nebraska 68588-0299, USA
}

(Received 18 September 2012; published 16 November 2012)

\begin{abstract}
The behavior of strong-field ionization rates of neutral atoms in the vicinity of multiphoton ionization thresholds is analyzed using formal collision theory. Our approach, which accounts nonperturbatively for effects of an intense laser field, shows that the ionization rates have a nearly constant behavior below and above each multiphoton threshold and that between such thresholds there are an apparently finite number of rapid oscillations due to resonances with laser-field-modified Rydberg states. This pattern is typical for any atomic target, as we illustrate specifically for hydrogen and neon atoms. The flat behavior of the ionization yield near multiphoton thresholds gives the appearance of an energy shift of the ionization thresholds, which have been postulated in a number of recent studies concerning diverse aspects of above-threshold ionization and high-harmonic generation of atoms. The flat behaviors of the rates near threshold exhibit only a rather weak dependence on the laser-field intensity. Other aspects of the near-threshold behavior of ionization rates and their dependence on the laser-field parameters are also discussed.
\end{abstract}

DOI: 10.1103/PhysRevA.86.053410

PACS number(s): $32.80 . \mathrm{Rm}, 32.80 . \mathrm{Fb}$

\section{INTRODUCTION}

A variety of processes in contemporary atomic and molecular physics (such as, e.g., electron impact excitation and ionization processes, cold atom collision processes, recombination processes, multiphoton ionization processes, etc.) have been studied intensively in the context of threshold laws [1]. While our focus in this paper is on threshold ionization of atoms in intense laser fields, we stress the universality of such threshold laws. In general, they define the energy dependence of the yield of a process in the vicinity of its threshold energy. A detailed theoretical description in the near-threshold region is often difficult, owing to the many intermediate states of interacting particles that must be taken into account. Threshold laws are particularly useful because they require only limited knowledge of the system in order to provide full information of the near-threshold energy behavior of the respective yields.

Theoretical analyses of the role of threshold laws in abovethreshold detachment (ATD) by strong laser fields have been carried out in many works [2-13]. While threshold effects in ATD are very well understood and confirmed by numerous calculations [2-13], similar recent studies devoted to abovethreshold ionization (ATI) or to high-harmonic generation (HHG) (a competing process) reach different conclusions (see, e.g., Refs. [14-16]). Thus, a main objective of this paper is to present a detailed analysis of the threshold behavior of multiphoton ionization with an emphasis on the role played by the intense laser field.

Note that our analysis is particularly relevant to experimental observations of striking ATI plateau enhancements for rare-gas atoms [2,17-21], i.e., that small changes in laser intensity can result in an order of magnitude increase of ATI electrons having a range of energies at the lower end of the ATI plateau. These experiments have stimulated a number of theoretical efforts aimed at identifying the origin of this effect. Especially intriguing was the discovery that similar enhancements are observed in the ATD process, in which atomic negative ions, instead of neutral atoms, are detached by a laser field [2-13]. Based on model calculations employing zero- and finite-range potentials, it was realized that the aforementioned enhancements stem from the behaviors of detachment yields at multiphoton thresholds, which are consistent with predictions of the Wigner threshold law [22]. In accordance with this law, it was demonstrated that the ATD plateau enhancements are sensitive to the initial state symmetry, with the most pronounced enhancements occurring for an initial state having $s$ or $p$ symmetry in the case of evenor odd-photon channel closings, respectively [6-9]. This was also supported by calculations of photoelectron fixed-angle energy spectra and angular distributions near the channel closings [13].

Although the threshold behavior of ATD spectra is very well understood owing to a large number of investigations of ATD plateau enhancements [2-13], a consistent interpretation of the threshold behavior of ATI and HHG processes for neutral atoms is far less firmly established. In the latter case, one must account for the role of the attractive Coulomb potential, including especially the Rydberg series converging to each multiphoton ionization threshold. In contrast to zero- and short-range potentials, which have a well-separated ionization continuum, the continuum in the case of a Coulomb potential is significantly modified by the laser-field-dressed Rydberg levels converging to it. This has been noted in a number of papers (see, e.g., Refs. [3,4,14-16,23,24]), nearly all of which put forth the idea of an effectively shifted ionization threshold [3,4,15,16,23,24]. In most of these works (cf. Refs. $[3,4,15,16,24])$ ad hoc physical arguments rather than detailed analytical explanations have been given for such a shift. In Ref. [23] the shift is related to the finite duration of the laser pulse; however, this explanation is not relevant to calculations or experiments involving long (monochromatic) laser pulses. In this paper we analyze the threshold behavior of multiphoton ionization rates from first principles for the case of an intense, monochromatic laser field.

In the context of this work, it is essential to stress that a very thorough analysis of the ionization spectra of neutral atoms treated in the so-called single-active electron (SAE) 
approximation has been performed in a series of papers by Potvliege and Shakeshaft [25-32]. These papers present very detailed analyses of resonances that are induced when the atomic ground state and some excited states are shifted into multiphoton resonances by an intense laser field. As discussed later on, the results presented in this paper and those of Refs. [23,25-32] show the generality of the predicted effects.

This paper is organized as follows. In Sec. II, we give a brief introduction of the quasistationary Floquet approach, which allows the treatment of time-periodic problems in quantum mechanics. In Sec. III, we present a theoretical description of threshold behavior in multiphoton ionization, taking into account an attractive Coulomb interaction in the final state. Our focus is on the role of the laser field, which is crucial for formation of an effective ionization threshold. In Sec. IV, numerical results for strong-field ionization of hydrogen and neon atoms are presented. In Sec. V, the respective yields are interpreted using the threshold law derived in Sec. III. In particular, we discuss the structure of the ionization spectra (Sec. V A), the energy shift of the ionization threshold (Secs. V B and V C), and the parity dependence of the ionization signal (Sec. V D). We summarize our results and discuss our conclusions in Sec. VI.

\section{FLOQUET THEORY}

We begin with a brief description of Floquet theory, which is a theoretical method that is useful when solving the time-dependent Schrödinger equation in the presence of a monochromatic laser field. In this case, the Hamiltonian $H(t)$ that describes the coupling of an atom to an external laser field $\mathcal{F}(t)$ can be written in the dipole velocity gauge as

$$
H(t)=H_{0}-\frac{i}{c} \mathcal{A}(t) \cdot \nabla,
$$

where $H_{0}$ is the atomic Hamiltonian in the single-active electron approximation,

$$
H_{0}=-\frac{\Delta}{2}+W(r),
$$

where $W(r)$ is the atomic potential (specified later on), and $\mathcal{A}(t)$ is the vector potential describing the laser field $\mathcal{A}(t)=$ $\mathcal{A}_{0} \hat{\boldsymbol{\varepsilon}} \sin \omega t$, where $\mathcal{A}_{0}=-c \mathcal{F}_{0} / \omega$. Here and throughout this paper, atomic units (a.u.) are used unless otherwise stated. Based on the periodicity of the Hamiltonian $H(t)=H(t+$ $2 \pi / \omega)$, we represent the quasistationary state of the system by the so-called Floquet ansatz [25],

$$
\Phi(\boldsymbol{r}, t)=e^{-i E t} \sum_{N} e^{-i N \omega t} \phi_{E}^{(N)}(\boldsymbol{r}),
$$

with $E$ being the quasienergy of the system and $\phi_{E}^{(N)}(\boldsymbol{r})$ the corresponding harmonic wave function. In Eq. (3), $N$ is interpreted as the net number of photons absorbed by the atom from the laser field. Substituting the above ansatz into the time-dependent Schrödinger equation, we find that the harmonics $\phi_{E}^{(N)}(\boldsymbol{r})$ satisfy the system of coupled equations,

$$
\left(H_{0}-E-N \omega+U_{p}\right) \phi_{E}^{(N)}+V_{+} \phi_{E}^{(N-1)}+V_{-} \phi_{E}^{(N+1)}=0,
$$

where $V_{ \pm}$are the Fourier coefficients of the dipole operator,

$$
V_{+} e^{-i \omega t}+V_{-} e^{i \omega t}=-\frac{i}{c} \mathcal{A}(t) \cdot \nabla,
$$

and where $U_{p}=\mathcal{F}_{0}^{2} / 4 \omega^{2}$ is the ponderomotive energy of the electron moving in the laser field. When solving the system of equations (4), appropriate boundary conditions for harmonics $\phi_{E}^{(N)}(\boldsymbol{r})$ must be enforced. The harmonics must be regular at small distances from the atomic center $(r \sim 0)$, whereas at large distances $(r \rightarrow \infty)$ they must satisfy the Siegert boundary conditions,

$$
\phi_{E}^{(N)}(\boldsymbol{r}) \sim \frac{e^{i k_{N} r}}{r},
$$

where $k_{N}$ is the wave number,

$$
k_{N}=\sqrt{2\left(E+N \omega-U_{p}\right)} .
$$

These boundary conditions cannot be satisfied unless the quasienergy is complex. Thus,

$$
E=E_{0}+\Delta-i \Gamma / 2,
$$

where $E_{0}$ is the initial energy level of the active electron, $\Delta$ is its ac Stark shift, and $\Gamma$ is its total detachment rate (for further details, see Refs. [8,9,13,25-32]). Note that for fixed parameters of the laser field, the amount of energy that has to be delivered in order to ionize a laser-dressed atom must be at least $E_{\min }=-\operatorname{Re} E+U_{p}$. This defines the minimum number of laser photons that are necessary to ionize the system as $N_{0}=1+\left[E_{\min } / \omega\right]$ (unless $E_{\min }=\left[E_{\min }\right]$, in which case $N_{0}=E_{\min } / \omega$ ). If the number of absorbed laser photons is such that $N \geqslant N_{0}$, or, equivalently $\operatorname{Re} E+N \omega-U_{p} \geqslant 0$, the $N$-photon ionization channel is open; otherwise, the $N$-photon channel is closed. For open channels one has outgoing spherical waves (6), while for closed channels one has exponentially damped waves. In actual computations [33], the infinite system of Eqs. (4) with boundary conditions (6) is solved by expanding the harmonic components on a discrete basis of complex radial Sturmian functions and spherical harmonics (after truncation of the number of basis functions). The numerical results are obtained by solving the resulting eigenvalue problem for the quasienergies and their associated Floquet states. Note that by changing the laser-field intensity (or the laser-field frequency) we can scan through the $N_{0}$-photon channel closing. This capability allows one to monitor the near-threshold behavior of the ionization rates, which we analyze next.

\section{NEAR-THRESHOLD BEHAVIOR OF MULTIPHOTON IONIZATION RATES}

A number of interesting properties concerning the energy dependence of cross sections near a reaction threshold can be derived using standard collision theory methods (see, for instance, Refs. [34,35]). As we have noted previously [8], these methods are difficult to develop for processes in a laser field, such as detachment or ionization, which is the main topic of our interest, since photons cannot be described by a wave function in position space. Thus in Ref. [8] we developed a formalism that allowed us to investigate the near-threshold behavior of detachment rates by using a stationary approach based on 
$R$-matrix theory $[36,37]$. We have shown in [8] that in the case of short-range potentials the $(N+s)$-detachment rates near the $N$-photon threshold exhibit Wigner-Baz' cusps [22,38]. In the following, we reformulate this theory to account for multiphoton ionization of neutral atoms when an attractive Coulomb potential in the final state must be taken into account.

In our approach [8], instead of introducing explicitly the initial state for the bound electron in the presence of a laser field, which leads to the Floquet equations (4) for the complex quasienergy, we introduce a source term $S_{N}(\boldsymbol{r})$ that is responsible for the outgoing flux in the open channels, thus mimicking their coupling with the initial state. This approach, even though not mathematically rigorous, allows us to treat the problem as quasistationary. The harmonic components of the Floquet state $\phi_{E}^{(N)}(\boldsymbol{r})$ thus satisfy the infinite set of coupled equations,

$$
\left(H_{0}-E-N \omega+U_{p}\right) \phi_{E}^{(N)}+V_{+} \phi_{E}^{(N-1)}+V_{-} \phi_{E}^{(N+1)}=S_{N},
$$

where $S_{N}(\boldsymbol{r})$ is the above-mentioned source term. The configuration space is divided into two regions, inside and outside a sphere of radius $r_{0}$, where $r_{0}$ is such that outside the sphere only the Coulomb force is assumed to act between the ionized electron and its parent ion. These assumptions are equivalent to those of $R$-matrix theory [34] and can be realized in the acceleration gauge provided $r_{0}>\alpha_{0}$ [39,40], where $\alpha_{0}=\sqrt{I} / \omega^{2}$ is the amplitude of electron quiver motion in the laser field.

A system of equations similar to (9), but homogeneous, was analyzed by Giusti-Suzor and Zoller [39] from the point of view of multichannel quantum defect theory (MQDT) [41,42]. For analysis of the threshold behavior we use a somewhat different, but equivalent, $R$-matrix method. A key point for the threshold analysis is that the electromagnetic interaction creates a dipole coupling between nondegenerate channels, decaying as $1 / r^{2}$, and a quadrupole coupling between degenerate channels, decaying as $1 / r^{3}$ [39]. This makes the diagonal Coulomb interaction $-1 / r$ dominant in each channel, and this interaction controls the threshold behavior of the ionization cross sections. Note that the stronger the laser field, the narrower the range of validity of the threshold laws (including the above-threshold Rydberg series). Thus, although determined by the Coulomb interaction, the threshold laws are strongly influenced by the laser field.

Consider now $M$ channels with energies $E+N \omega-U_{p}$, where $N=1,2, \ldots, M$. The solution to Eq. (9) inside the $R$ matrix sphere can be represented in its most general form as [8]

$$
\phi_{E}^{(\mathrm{in})}(\boldsymbol{r})=f(\boldsymbol{r})+u(\boldsymbol{r}) A .
$$

Here, $\phi_{E}^{(\mathrm{in})}$ is the solution vector with components $\phi_{E}^{(N)}$ $(N=1, \ldots, M), u$ is the solution matrix of the homogeneous equations with rows corresponding to different channels and columns corresponding to different linearly independent solutions, $f$ is a column vector comprising particular solutions of the inhomogeneous equations (9), and $A$ is a vector of arbitrary coefficients. Both $f$ and $u$ are determined by boundary conditions at the origin that are independent of energy; therefore they can be considered as entire functions of $E$. Outside the $R$-matrix sphere there are only outgoing waves in open channels, and exponentially decaying solutions in closed channels, so that the solution vector is given by

$$
\phi_{E}^{\text {(out) }}(\boldsymbol{r})=O T,
$$

where $O$ is a diagonal matrix of outgoing solutions and $T$ is a column vector defining their amplitudes. For an attractive Coulomb field the components of $O$ have the following asymptotic form:

$$
O_{N} \sim \frac{1}{\sqrt{v_{N}}} \exp \left\{i\left[k_{N} r-l \pi / 2-\eta_{N} \ln \left(2 k_{N} r\right)+\eta_{N, l}\right]\right\},
$$

where $v_{N}=k_{N}$ is the electron velocity in the $N$ th channel, $\eta_{N}=-1 / k_{N}$, and $\eta_{N, l}$ is the Coulomb phase, $\eta_{N, l}=$ $\arg \Gamma\left(l+1+i \eta_{N}\right)$. The normalization of the solution $O_{N}$ in Eq. (12) corresponds to unit flux density in the outgoing channel. These solutions are appropriate for an atomic potential such that $W(r) \sim-1 / r$ at large distances from the atomic center, as is the case for the $\mathrm{H}$ and $\mathrm{Ne}$ atoms treated in Sec. IV. The vector $T$ in Eq. (11) comprises the ionization amplitudes $T_{N}$ that determine the $N$-photon ionization rates, where the differential rate $d w_{N}$ in the direction $\hat{\boldsymbol{r}}$ is given by

$$
d w_{N}=\left|T_{N}\right|^{2}\left|Y_{l m}(\hat{\boldsymbol{r}})\right|^{2} d \hat{\boldsymbol{r}},
$$

where $l$ and $m$ are the electron angular momentum and its projection in the $N$ th channel. Upon matching the inner $\phi_{E}^{(\text {in) }}(\boldsymbol{r})$ and outer $\phi_{E}^{\text {(out) }}(\boldsymbol{r})$ wave functions and their derivatives at $r=$ $r_{0}$, the vector of ionization amplitudes is given by

$$
T=\left.(L O)^{-1}(L f)\right|_{r=r_{0}},
$$

where

$$
L=R \frac{d}{d r}-1, \quad R=\left.u\left(\frac{d u}{d r}\right)^{-1}\right|_{r=r_{0}} .
$$

In order to study the threshold behaviors of the ionization rates, we need now to exploit the analytic properties of the matrix elements $O$ as a function of the channel energy $k_{N}^{2} / 2$ [see Eq. (7)] in a way similar to that of our previous studies on multiphoton detachment [8].

For ionization in the presence of the long-range Coulomb potential outside of the $R$-matrix sphere, the general solution $O_{N}$ can be written as a linear combination of regular and irregular Coulomb functions, with asymptotic behavior as given in Eq. (12). Specifically, one can write [35]

$$
O_{N}=\xi_{l}(k r) C_{l}^{-1}(k)(k r)^{-l-1 / 2}+i \rho_{l}(k r) C_{l}(k)(k r)^{l+1 / 2},
$$

where $\xi_{l}(k r)$ and $\rho_{l}(k r)$ are real analytic functions of $k r$, where $k$ and $l$ are the linear and angular momenta in the $N$ th channel, and $C_{l} \equiv C_{l}(k)$ is a function of $l$ and $k$ such that

$$
\begin{gathered}
C_{0}=\left(\frac{2 \pi \eta}{e^{2 \pi \eta}-1}\right)^{1 / 2}, \\
C_{l}=\frac{2^{l} C_{0}}{(2 l+1) !}\left[\left(1^{2}+\eta^{2}\right)\left(2^{2}+\eta^{2}\right) \ldots\left(l^{2}+\eta^{2}\right)\right]^{1 / 2},
\end{gathered}
$$

where $\eta=-k^{-1}$. Substituting (16) into (14) gives

$$
T=C_{l} k^{l+1 / 2}\left(M-i C_{l}^{2} k^{2 l+1}\right)^{-1} g,
$$


where

$$
\begin{gathered}
M=-\left.(L \rho)^{-1}(L \xi)\right|_{r=r_{0}}, \\
g=-\left.(L \rho)^{-1}(L f)\right|_{r=r_{0}} .
\end{gathered}
$$

Note that on the right side of Eq. (16), we have omitted the subscripts $N$ in order to simplify the notation. In Eq. (19), $C_{l} k^{l+1 / 2}$ and $C_{l}^{2} k^{2 l+1}$ are diagonal matrices in which $l$ and $k$ take appropriate values in each channel $N$. The vector $T$ in Eq. (19) reduces to that for multiphoton detachment [cf. Eq. (30) of Ref. [8]] upon neglecting $C_{l}$ and introducing appropriate changes in the definitions of $M$ (20) and $g$ (21). As in that case, we treat both the matrix $M$ and the vector $g$ as analytic functions of energy.

Let us analyze the behavior of the $(N+s)$-photon ionization rate at the $N$-photon ionization threshold. For simplicity, we denote the $N$-photon channel as channel number 1 , and the $(N+s)$-photon channel as channel 2. Near the $N$-photon channel threshold, $k_{1}^{2}$ is small but can be either positive or negative [cf. Eq. (7)]. In particular, for $k_{1}^{2}>0$ the $N$ photon ionization channel is open; hence the intensity is below that which closes the $N$-photon threshold. For $k_{1}^{2}<$ 0 , the situation is reversed. In the following, we consider channel closings in the direction of increasing laser-field intensity. Thus we refer to the case when the $N$-photon threshold is approached "from below" for $k_{1}^{2}>0$ and "from above" for $k_{1}^{2}<0$. With increasing laser-field intensity, the ponderomotive energy $U_{p}$ increases, thus motivating one to analyze the behavior of multiphoton ionization rates when passing through a channel closing. With this in mind, we derive from Eq. (19) the ionization amplitude in the $i$ th channel $(i=1,2)$ :

$$
T_{i}=C_{l_{i}} k_{i}^{l_{i}+1 / 2} \frac{\left(M_{j j}-i C_{l_{j}}^{2} k_{j}^{2 l_{j}+1}\right) g_{i}-M_{12} g_{j}}{\operatorname{det} M-i M_{i i} C_{l_{j}}^{2} k_{j}^{2 l_{j}+1}-i M_{j j} C_{l_{i}}^{2} k_{i}^{2 l_{i}+1}-C_{l_{1}}^{2} k_{1}^{2 l_{1}+1} C_{l_{2}}^{2} k_{2}^{2 l_{2}+1}},
$$

where $j=2(1)$ when $i=1(2)$ and where $\operatorname{det} M=M_{11} M_{22}-$ $M_{12}^{2}$. Using (22) we can analyze the energy variations of the ionization rates in both channels in the limit that $k_{1}^{2}$ is small. For this purpose, we introduce a new variable,

$$
s_{i}=C_{l_{i}}^{2} k_{i}^{2 l_{i}+1}
$$

(for $i=1,2$ ) so that Eq. (22) becomes

$$
\left|T_{i}\right|^{2}=s_{i}\left|\frac{\left(M_{j j}-i s_{j}\right) g_{i}-M_{12} g_{j}}{\operatorname{det} M-i M_{i i} s_{j}-i M_{j j} s_{i}-s_{1} s_{2}}\right|^{2} .
$$

In addition, we note that $\left|T_{1}\right|^{2}=0$ above the $N$-photon threshold. According to Eq. (13), Eq. (24) determines the threshold behavior of the partial ionization rates in the $i$ th channel.

Consider first the case when the ponderomotive shift $U_{p}$ decreases such that the $N$-photon ionization threshold is approached from above. In this case, $k_{1}^{2} \rightarrow 0^{-}$, and so we define $k_{1}=i \kappa$, which means also that $\eta_{1}=i / \kappa$. Based on Eqs. (17) and (18), one can show that in the limit that $\kappa \rightarrow 0$,

$$
s_{1} \sim \pi\left[\frac{2^{l_{1}}}{\left(2 l_{1}+1\right) !}\right]^{2}\left[1+i \cot \left(\frac{\pi}{\kappa}\right)\right] .
$$

The threshold behavior in the $(N+s)$-photon channel is therefore governed by

$$
\left|T_{2}\right|^{2} \sim s_{2}\left|\frac{a+b \cot \left(\frac{\pi}{\kappa}\right)}{\alpha+\beta \cot \left(\frac{\pi}{\kappa}\right)}\right|^{2},
$$

where for completeness we also specify the coefficients:

$$
\begin{gathered}
a=M_{11} g_{2}-M_{12} g_{1}-i b, \quad b=c_{1} g_{2}, \\
\alpha=\operatorname{det} M-i\left(s_{2} M_{11}+\beta\right), \quad \beta=c_{1}\left(M_{22}-i s_{2}\right),
\end{gathered}
$$

where

$$
c_{1}=\pi\left[\frac{2^{l_{1}}}{\left(2 l_{1}+1\right) !}\right]^{2} .
$$

One sees from Eqs. (26) and (27) that the rate for ionization in the $(N+s)$-photon channel is trivial if $a=b=0$, which is equivalent to the condition that $g_{1}=g_{2}=0$. This means that there is no coupling with the initial channel, and so there is no laser field driving ionization.

Above the $N$-photon channel closing, the $(N+s)$-photon ionization rate exhibits an oscillatory behavior, governed by the function $\varphi(\kappa) \equiv\left|\frac{a+b \cot \left(\frac{\pi}{k}\right)}{\alpha+\beta \cot \left(\frac{\pi}{\kappa}\right)}\right|^{2}$ [cf. Eq. (26)], which is illustrated in Fig. 1 for arbitrarily chosen coefficients $a, b, \alpha, \beta$ (that are specified in the figure caption). The function $\varphi(\kappa)$ is maximal (minimal) for argument values $\kappa=\kappa_{m}^{+}\left(\kappa_{m}^{-}\right)$, where

$$
\frac{\pi}{\kappa_{m}^{ \pm}}=m \pi+\operatorname{arccot}\left[\operatorname{Re}\left(-\frac{\alpha}{\beta}\right)+\frac{\operatorname{Im} \delta \pm|\delta|}{\operatorname{Re} \delta} \operatorname{Im}\left(-\frac{\alpha}{\beta}\right)\right] \text {, }
$$

in which we have introduced the parameter $\delta$, defined by

$$
\delta=b\left(a-b \frac{\alpha}{\beta}\right)-i \frac{\left|a-b \frac{\alpha}{\beta}\right|^{2}}{2 \operatorname{Im}\left(-\frac{\alpha}{\beta}\right)},
$$

and where $m$ is an integer such that $\kappa_{m}^{ \pm} \geqslant 0$. The extrema $\varphi^{ \pm} \equiv \varphi\left(\kappa_{m}^{ \pm}\right)$are given by

$$
\varphi^{ \pm}=\frac{1}{|\beta|^{2}}\left[b^{2} \pm \frac{|\delta| \mp \operatorname{Im} \delta}{\operatorname{Im}\left(-\frac{\alpha}{\beta}\right)}\right],
$$

in which we have replaced $\left|\operatorname{Im}\left(-\frac{\alpha}{\beta}\right)\right|$ by $\operatorname{Im}\left(-\frac{\alpha}{\beta}\right)$ since it follows from the definitions of $\alpha$ and $\beta$ [Eq. (28)] that $\operatorname{Im}\left(-\frac{\alpha}{\beta}\right)=1+\frac{s_{2} M_{12}^{2}}{c_{1}\left(s_{2}^{2}+M_{22}^{2}\right)}>0$. One can understand from the above analysis as well as from Fig. 1 that the heights of 


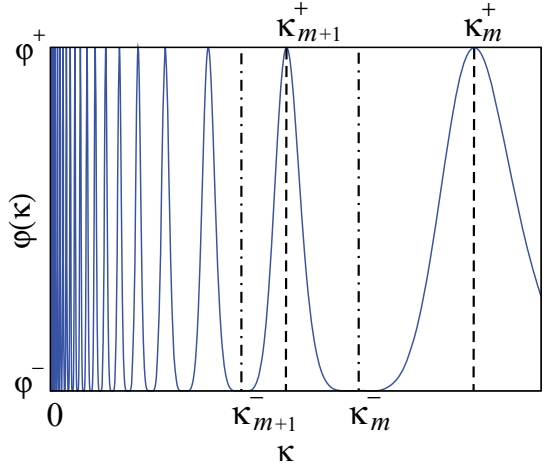

FIG. 1. (Color online) Plot of $\varphi(\kappa) \equiv\left|\frac{a+b \cot \left(\frac{\pi}{\kappa}\right)}{\alpha+\beta \cot \left(\frac{\pi}{\kappa}\right)}\right|^{2}$ vs the energy parameter $\kappa$ for arbitrarily chosen coefficients: $a=1-i, b=$ $1, \alpha=2-i$, and $\beta=1+3 i$. The function $\varphi(\kappa)$ is responsible for the oscillatory behavior of the $(N+s)$-photon ionization rate $\left|T_{2}\right|^{2}$ above the closing of the $N$-photon threshold at $\kappa=0$ [cf. Eq. (26)]. Note that for fixed photon frequency, increasing $\kappa$ corresponds to increasing laser intensity [cf. Eq. (7) and note that $k_{N} \equiv k_{1} \equiv i \kappa$ ]. The values of the extrema $\varphi^{ \pm} \equiv \varphi\left(\kappa_{m}^{ \pm}\right)$are given in Eq. (32) and the locations of two maxima, $\kappa_{m}^{+}$and $\kappa_{m+1}^{+}$, and two minima, $\kappa_{m}^{-}$and $\kappa_{m+1}^{-}$, are indicated respectively by the dashed and dot-dashed vertical lines.

these oscillations do not change with $\kappa$, as $\kappa$ decreases or, in other words, as the laser-field intensity $I$ decreases. However, the frequency of these oscillations in the ionization rates in the $(N+s)$-photon channel increases when approaching the $N$-photon threshold from above. These oscillations reflect the fact that the $(N+s)$-photon ionization channel interacts with successive $N$-photon Rydberg state resonances as the laserfield intensity decreases. These Coulomb field resonances become more stable against ionization when approaching the threshold, as their width drops to zero. Thus, resonances with infinitely many Rydberg states should be observed when decreasing the ponderomotive shift toward the threshold. As we illustrate later on, however, this expectation is not realized in our results. The reason is that the above-threshold oscillations in the ionization signal shown in Fig. 1 can be strongly suppressed by the factor $s_{2}$ [cf. Eq. (26)], which depends on the orbital angular momentum $l_{2}$ and energy $k_{2}$ in the $(N+s)$-photon channel. We analyze this behavior of $s_{2}$ in Sec. $\mathrm{V}$.

Below the $N$-photon channel threshold, when $k_{1}^{2} \rightarrow 0^{+}, s_{1}$ tends to a nonzero constant. This follows from the definition of the matrix coefficients (17) and (18) when analyzed in the respective limit; specifically, $s_{1} \sim 2 c_{1}$ [cf. Eq. (29)] for $k_{1}^{2} \rightarrow$ $0^{+}$, while $s_{2}$ is smooth across the threshold (cf. Sec. V). In this case, we conclude from Eq. (24) that both the $N$-photon and $(N+s)$-photon ionization rates are finite and constant with respect to the electron energy in the closing channel. In particular, we find that the $(N+s)$-photon ionization rate below the $N$-photon threshold behaves as

$$
\left|T_{2}\right|^{2} \sim s_{2}\left|\frac{a-i b}{\alpha-i \beta}\right|^{2}
$$

with the coefficients $a, b, \alpha$, and $\beta$ defined by Eqs. (27) and (28).

Comparing Eq. (26) for $\kappa \rightarrow 0$ with Eq. (33), we see that the ionization rate into the higher $(N+s)$-photon channel exhibits a jump at the $N$-photon threshold. In scattering this is also the case [35,37]. Moreover, it was proved in Refs. [35,37] that, when averaged over oscillations, the scattering cross sections for each of the channels that are open below the threshold of a closed channel show downward steps at the threshold (beyond which the closed channel becomes open). These steps are such that the total scattering cross section becomes continuous across this threshold [35,37]. In order to calculate the oscillation-averaged ionization rate in the $(N+s)$-photon channel above the $N$-photon threshold, it is enough to calculate this average over one branch of $\cot (\pi / \kappa)$ [cf. Eq. (26)]. To do so, we introduce the new variable $x=\cot (\pi / \kappa)$ and perform the integration over $x$ from $-\infty$ to $+\infty$ using the residue theorem, closing the integration contour in the upper half-plane. The poles enclosed by this integration contour are at $x=i$ and $x=-\frac{\alpha}{\beta}$, since in our case $\operatorname{Im}\left(-\frac{\alpha}{\beta}\right)>0$. Calculating the residues at each of the poles, we obtain the following oscillation-averaged ionization rate in the $(N+s)$-photon open channel above the closed $N$-photon channel:

$$
\overline{\left|T_{2}\right|^{2}} \sim s_{2}\left|\frac{a-i b}{\alpha-i \beta}\right|^{2}+\frac{s_{2}}{\operatorname{Im}\left(-\frac{\alpha}{\beta}\right)}\left|\frac{a-b \frac{\alpha}{\beta}}{\alpha-i \beta}\right|^{2} .
$$

It follows from this equation that the oscillation-averaged partial ionization rate $\overline{\left|T_{2}\right|^{2}}$ for an $(N+s)$-ionization channel exhibits, in general, an upward step when crossing the $N$ photon threshold as the laser-field intensity increases and closes the $N$-photon ionization channel. This agrees with the collision theory predictions [35,37], and may be related to the experimentally observed ATI plateau enhancements in rare-gas atoms [2,17-21]. Specifically, when one partial cross section becomes closed, the remaining open partial cross sections increase so that the total cross section is continuous. Since the partial cross sections that become closed typically have much greater magnitudes than the open partial cross sections on the ATI plateau, it should not be surprising that increases of the latter may be quite large. Numerical analyses of strong-field detachment of negative ions indeed found that at the intensity at which the ATD enhancement occurs, the portion of the partial cross sections in higher photon detachment channels (that belong to the low- and mid-energy part of the rescattering plateau) increase tremendously. Even though we have no access to numerical data on partial ionization rates for neutral atoms, our analytical analysis is applicable for any $(N+s)$-photon channel, including the direct and rescattering parts of the ATI amplitude. Below, we discuss the consequences of the threshold behaviors predicted by our general analysis for various properties of ATI spectra.

It follows from Eqs. (26) and (30) that the partial ionization rates in the open channels vary rapidly if $\cot \left(\frac{\pi}{\kappa}\right) \simeq \operatorname{Re}\left(-\frac{\alpha}{\beta}\right)$, which determines the positions of resonances on the energy scale. [For details, see Fig. 1 and the discussion above of $\varphi(\kappa)$.] This condition indicates that the resonances are displaced with respect to the bound-state energy levels in the Coulomb field, given by $\cot \left(\frac{\pi}{\kappa}\right)= \pm \infty$. However, this shift is expected since the system is embedded in the laser field, and so only resonances with the field-dressed Rydberg states should be observed. 
Consider the limit when $c_{1} \ll 1$. From Eq. (29) we see that $c_{1}$ is a monotonically decreasing function of $l_{1}$, the orbital angular momentum of the active electron after its absorption of $N$ laser photons. Remarkably, $c_{1}$ becomes negligibly small for only moderate values of $l_{1}$ : for $l_{1}=5, c_{1} \sim 10^{-12}$, and for $l_{1}=6, c_{1} \sim 10^{-16}$. In fact, $c_{1}$ decreases very rapidly even for small values of $l_{1}$ : The difference in the values of $c_{1}$ for $s$ and $p$ electrons is nearly an order of magnitude. For $c_{1} \ll 1$, the $N$-photon ionization rate below its threshold is small. In addition, it follows from Eqs. (26), (27), (28), and (33) that the $(N+s)$-photon ionization rates above and below the $N$-photon threshold have nearly the same value, $\left|T_{2}\right|^{2} \sim s_{2}\left(M_{11} g_{2}-M_{12} g_{1}\right)^{2} /\left[(\operatorname{det} M)^{2}+\left(s_{2} M_{11}\right)^{2}\right]$, which is constant with respect to the electron energy in the closing channel. Therefore, in order to observe pronounced oscillations of the partial ionization rates in higher photon channels (as found numerically in results of Refs. [25,26,28-32] as well as in our results in Sec. IV), the outer electron in the $N$-photon channel must have a rather low orbital angular momentum, preferably $l_{1}=0$ or 1 .

Consider next the second term in Eq. (34). It follows from our previous discussion that for small values of $c_{1}, \operatorname{Im}\left(-\frac{\alpha}{\beta}\right) \sim$ $\frac{1}{c_{1}}$. From Eqs. (27) and (28), one sees that $a-b \frac{\alpha}{\beta}$ does not depend on $c_{1}$, whereas $\alpha-i \beta=\operatorname{det} M-i s_{2} M_{11}-2 i c_{1}\left(M_{22}-\right.$ $i s_{2}$ ) is a nonzero complex number in the limit $c_{1} \rightarrow 0$. Hence, one deduces that the second term in Eq. (34) is proportional to the coefficient $c_{1}$ and consequently decreases with decreasing $c_{1}$. We conclude, therefore, that when the active electron, after absorbing $N$ laser photons, attains a moderately large orbital angular momentum, only a very small jump in the resonanceaveraged partial ionization rates of higher $(N+s)$-photon channels should be observed at the closing of the $N$-photon threshold, in agreement with the discussion in the previous paragraph. Consequently, essentially no enhancement of the ATI energy spectrum should be observed if the orbital angular momentum $l_{1}$ in the closing channel is high. From Eq. (29), one sees that such an enhancement would be most pronounced for those $N$-photon channels that result in an $s$ or a $p$ electron in the final state, for which $c_{1}$ is largest. This threshold-related feature should be observed in general for any atomic target [so long as the single-active electron approximation is valid], thus proving analytically that the experimentally observed ATI enhancements are a single electron effect, in agreement with the numerical findings of solutions of the time-dependent Schrödinger equation carried out in the SAE approximation that confirmed the experimental observations $[19,20,43,44]$.

One can anticipate that all features concerning the nearthreshold behavior of partial ionization rates, which we have discussed above, will be reflected in the total ionization rate. This is investigated next in Sec. IV, where we present our numerical results for ionization of $\mathrm{H}$ and $\mathrm{Ne}$ atoms.

\section{IONIZATION OF H AND NE}

Consider first the ionization of a hydrogen atom by a monochromatic, linearly polarized laser field with $\lambda=$ $1000 \mathrm{~nm}(\omega=0.0456$ a.u. $)$. For a weak laser field, at least 11 photons are needed for ionization to occur. However, the minimum number of photons required for ionization increases

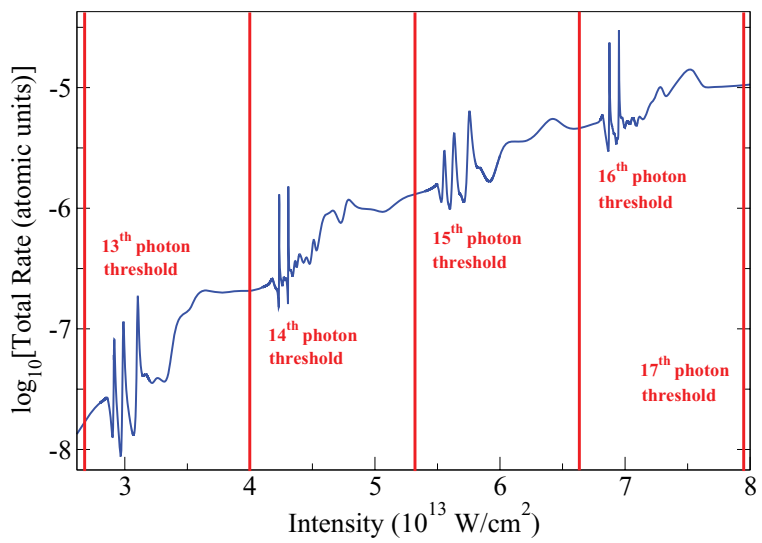

FIG. 2. (Color online) Total ionization rate vs laser-field intensity for ionization of the hydrogen atom by a monochromatic, linearly polarized laser field of wavelength $\lambda=1000 \mathrm{~nm}$. Vertical solid lines mark (from left to right, respectively) the 13th, 14th, 15th, 16th, and 17 th photon thresholds.

with increasing laser-field intensity (or with decreasing laserfield frequency). Thus, by varying the laser-field intensity, we pass through sequential channel closings. In Fig. 2, we present the total ionization rate $\Gamma=-2 \operatorname{Im} E$, as a function of the laser-field intensity $I$, traversing the 13th, 14th, 15 th, 16 th, and 17th photon thresholds, as marked in the figure. We observe four distinct series of peaks, shown in more detail in Fig. 3.

Consider next the ionization of a neon atom, which in contrast to hydrogen with its $s$-valence electron, has a $p$-valence electron. We describe neon within the SAE approximation, using the model potential of Tong and Lin [45],

$$
W(r)=-\frac{Z_{c}+a_{1} e^{-a_{2} r}+a_{3} r e^{-a_{4} r}+a_{5} e^{-a_{6} r}}{r} .
$$

Here, $Z_{c}=1$ is the charge seen by the active electron at large distances from the nucleus, and the coefficients

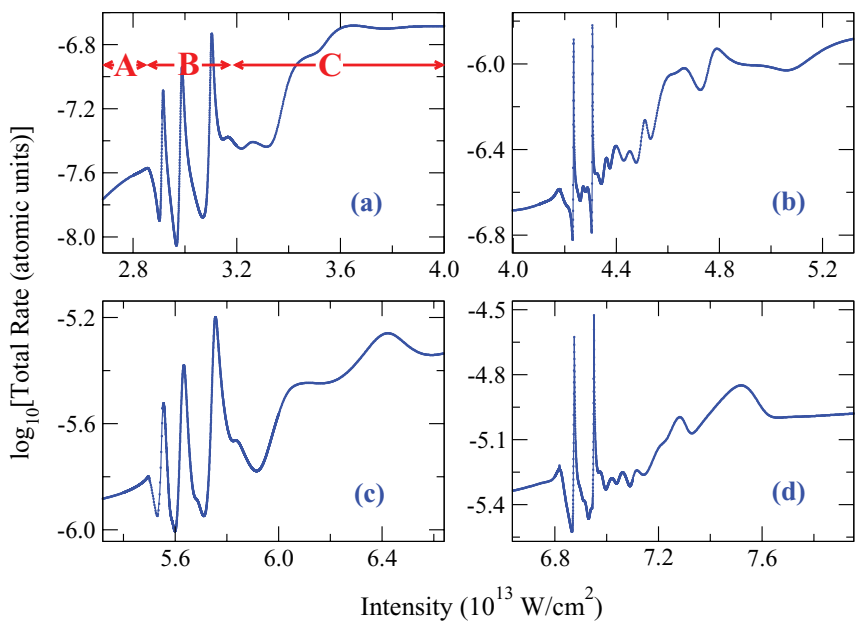

FIG. 3. (Color online) Total ionization rate for the $\mathrm{H}$ atom, as in Fig. 2, on an enlarged scale in four intensity regions between (a) the 13th and 14th photon thresholds; (b) the 14th and 15th photon thresholds; (c) the 15th and 16th photon thresholds; and (d) the 16th and 17th photon thresholds. In panel (a), the three regions marked as "A," "B," and "C" are discussed in Sec. V A. 


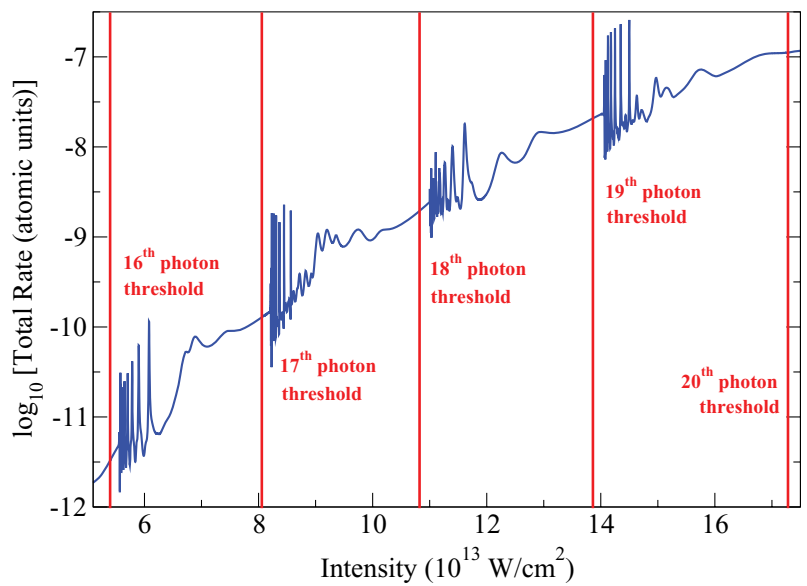

FIG. 4. (Color online) Total ionization rate for neon ionized by linearly polarized, $800 \mathrm{~nm}$ laser light plotted vs laser-light intensity $I$. Vertical solid lines mark sequential ionization thresholds, as indicated in the figure.

$a_{i}(i=1, \ldots, 6)$ have been fitted to a numerical potential obtained from density functional theory. From Ref. [45], $a_{1}=$ 8.069, $a_{2}=2.148, a_{3}=-3.570, a_{4}=1.986, a_{5}=0.931$, and $a_{6}=0.602$. For this model potential, the calculated binding energy of the ground $p$ state is $E_{0}=-0.793$ a.u., which is in very good agreement with the experimental value.

The total rate for ionization of the neon atom, described by the model potential (35), by linearly polarized, $800 \mathrm{~nm}$ light $(\omega=0.057$ a.u. $)$ is shown in Fig. 4. For a weak $800 \mathrm{~nm}$ laser field, at least 14 photons are necessary to ionize the neon atom. This number increases every time the ponderomotive energy increases by approximately the energy of one laser photon. The total ionization rate is calculated for a range of laser-field intensities such that we pass through the 16th, 17th, 18th, 19 th, and 20th photon thresholds. We mark these multiphoton thresholds by the vertical solid lines in Fig. 4.

Comparing the results for hydrogen (Fig. 2) and neon (Fig. 4), we observe very similar behaviors of the ionization rates over an energy range corresponding to one photon energy. Specifically, a threefold pattern in the energy dependence of the total ionization rates is observed in each case. First, below each ionization threshold the total ionization rate is constant or changes very slowly with intensity [e.g., cf. region " $C$ " in Fig. 3(a)]. Well above each ionization threshold one observes a series of sharp (high-frequency) oscillations corresponding to resonances with dressed excited states [e.g., cf. region "B" in Fig. 3(a)]. However, instead of an infinite number of resonant oscillations, one observes only a finite number. In addition, their heights tend to vanish as one approaches the next threshold from above, thus saturating before the threshold is attained [e.g., cf. region " $A$ " in Fig. 3(a)]. In the remainder of this paper, we analyze this threshold-related behavior of strong-field ionization and provide explanations for the numerically calculated structures.

\section{ANALYSIS AND DISCUSSION}

In this section we provide a detailed analysis of the structural patterns observed in our calculated ionization rates shown in Figs. 2-4. These patterns appear to be quite general features for both $s$-valence electron atoms $(\mathrm{H})$ and $p$-valence electron atoms $(\mathrm{Ne})$, at least when ionization is treated within the single-active electron approach used throughout this paper. In particular, the threefold pattern of ionization spectra was also observed in the numerical results of Ref. [23] for ionization by a weak pulsed laser field and in Refs. [31,32] for ionization by an intense monochromatic laser field, thus both confirming our findings and indicating the robustness of this pattern in ATI spectra. While the focus of our analysis is on the structural patterns of the ionization spectra, our analysis also sheds light on the concept of an effective ionization threshold as well as on the influence of the initial state parity of the active electron.

\section{A. The threefold spectral pattern}

To make our analysis more systematic, we focus on Fig. 3 in each of whose panels we present the total ionization yield over the range of laser-field intensities between the $N$ - and the $(N+1)$-photon thresholds, i.e., over which the ponderomotive potential changes by approximately the energy of one photon. The total ionization yields traverse three distinct regions as the ponderomotive shift changes, shifting the ground state into resonance with different atomic levels. The structural formations in the total ionization yield spectra depend largely on the orbital angular momenta of the resonant levels. Going in the direction of increasing laser-field intensity, one observes three regions: "A" the total ionization rate passes through the $N$-photon threshold smoothly, remaining nearly flat or at most slowly increasing; " $B$ " the ionization rate exhibits very pronounced oscillatory behavior; and " $C$ " it shows a broad hilly structure, which then becomes flat again before the $(N+1)$-photon threshold is reached. These regions are marked in panel (a) of Fig. 3. In the following, we give a general explanation of the origin of these three distinct structural regions.

\section{Region of resonances with broad, low-energy excited states}

The broad structures in region " $\mathrm{C}$ " originate from $N$-photon resonances with the lowest possible $n l$ excited states. Excited states having low principal quantum number $n$ are shifted most by the laser field. Moreover, low $l$ states $(l=0, \ldots, n-1)$ are less stable against ionization. In a laser field, these states acquire widths that grow with increasing laser-field strength. Eventually, they begin to overlap, forming a broad quasicontinuum, which is similar to the ones proposed by Faria et al. [4] and by Frolov et al. [15], as we discuss later. Thus, the broad structures that we see in region " $C$ " are the fingerprints of an isolated quasicontinuum formed by low-energy excited states. At the same time, we observe a pronounced increase of the total ionization rate for odd symmetry states in the closing channels. This can be seen particularly well for hydrogen in Fig. 3: While in panels (a) and (c) we observe a pronounced steplike feature in region " $C$ " following an odd-photon channel closing, this feature is less pronounced in panels (b) and (d) following an even-photon channel closing. As the laser intensity increases, this resonant structure disappears and the total ionization rate becomes 
constant below the $(N+1)$-photon threshold, in agreement with the predictions of Sec. III.

\section{Region of resonances with narrow, high-energy excited states}

The pronounced oscillatory structures appearing in region "B" originate from resonances with higher-energy excited $n l$ states, including, in particular, higher $l$ states than those contributing to the structures in region "C." Resonances with these more stable states results in rapid oscillations in the ionization yield as the intensity decreases, as predicted in Sec. III. Moreover, the positions of these resonances are shifted less by the laser field owing to the fact that the energy shift of excited states, when dressed by the laser field, decreases with increasing $n$. Consequently, the resonances observed in region "B" are similar to those of the field-free Coulomb spectrum.

By way of illustration, consider the resonances between the 15th and the 16th photon thresholds in ionization of hydrogen by a $1 \mu \mathrm{m}$ laser field [Fig. 3(c)]. The 15th photon threshold is closed at a laser intensity of $I_{\text {th }}=5.319 \times$ $10^{13} \mathrm{~W} / \mathrm{cm}^{2}$, in which case the corresponding quasienergy is $E\left(I_{\text {th }}\right)=\left(-0.50178744-1.3083087 \times 10^{-6} i\right)$ a.u. One sees in Fig. 3(c) three pronounced resonances above this channel closing as the laser-field intensity increases. In Table I, we give the values of the quasienergies corresponding to these three resonances and the intensities at which they occur. According to an MQDT analysis [39], the ac Stark shift for high $n$ decreases as $n^{-3}$. Assuming therefore that high $n l$ states do not shift with respect to the threshold energy, we can set the difference of the Stark shift and the ponderomotive potential energies equal to the excited state energy, $E_{n^{*}}=-1 / 2 n^{* 2}$, so that

$$
E_{n^{*}} \simeq \operatorname{Re} E(I)-U_{p}(I)-\left[\operatorname{Re} E\left(I_{\mathrm{th}}\right)-U_{p}\left(I_{\mathrm{th}}\right)\right] .
$$

Based on this equation, the principal quantum number $n$ labeling the Rydberg state that undergoes the 15-photon resonance with the $1 s$ state can be estimated; these estimates are given in Table I. In fact, there we give two values: the principal quantum number $n$ and the effective quantum number $n^{*}$, where $n^{*}$ is obtained from Eq. (36) and $n=\left[n^{*}\right]+1$. The difference between the two indicates that the positions of the resonances are shifted with respect to the pure Coulomb resonances, as predicted in Sec. III, i.e., the Rydberg states are dressed by the laser field, and so the difference of $n^{*}$ from $n$ accounts for this effect. The dressing by the laser field is particularly noticeable for lower-energy excited states. Indeed,

TABLE I. Positions and widths of resonances between the 15-photon and 16-photon ionization thresholds when a hydrogen atom is exposed to a $1000 \mathrm{~nm}$ linearly polarized laser field. The 15-photon channel closing occurs at the laser-field intensity $I_{\mathrm{th}}=5.319 \times 10^{13} \mathrm{~W} / \mathrm{cm}^{2}$, in which case $E\left(I_{\mathrm{th}}\right)=(-0.50178744-$ $\left.1.3083087 \times 10^{-6} i\right)$ a.u.

\begin{tabular}{lccc}
\hline \hline $\begin{array}{c}\text { Intensity } \\
\left(\mathrm{W} / \mathrm{cm}^{2}\right)\end{array}$ & $\begin{array}{c}\operatorname{Re} E \\
(\text { a.u. }\end{array}$ & $\begin{array}{c}\Gamma / 2=\operatorname{Im} E \\
\text { (a.u.) }\end{array}$ & $\begin{array}{c}\text { Rydberg level } \\
n\left(n^{*}\right)\end{array}$ \\
\hline $5.555 \times 10^{13}$ & -0.50186980 & $2.9898182 \times 10^{-6}$ & $8(7.82)$ \\
$5.634 \times 10^{13}$ & -0.50189707 & $4.1650419 \times 10^{-6}$ & $7(6.77)$ \\
$5.756 \times 10^{13}$ & -0.50193943 & $6.3344807 \times 10^{-6}$ & $6(5.75)$ \\
\hline \hline
\end{tabular}

one sees from Table I that the lower the value of $n$, the larger the difference $n-n^{*}$, so that the lower $n$ is, the greater the shift away from the 15-photon threshold, as has been shown also in Refs. [31,32]. This may also indicate that the broad double-peak structure appearing at higher intensity is due to the resonances with the next lower excited states, having principal quantum numbers $n=5$ and $n=4$, whereas resonances with the still lower-lying excited states $(n=3$ and $n=2)$ are not observed before the 16th photon threshold is reached.

\section{Smooth region in which high angular momentum resonances are suppressed}

As one approaches the $N$-photon threshold, one enters region "A," which is characterized by a rather flat dependence of the total ionization rate on the laser-field intensity. In principle, in this region one should observe resonances with even higher-energy Rydberg states than in region "B." This is not the case, however. The numerical results in Figs. 2 and 3, as well as those of Table I, show that the heights of the resonant spikes vanish with increasing principal quantum number $n$ of the resonant Rydberg states. As mentioned in Sec. III, the oscillatory behavior of the ionization rate in the $(N+s)$-photon channel above the $N$-photon threshold can be efficiently suppressed by the factor $s_{2}$. Note that for a fixed $(N+s)$-photon channel with a fixed orbital angular momentum $l_{2}, s_{2}$ remains nearly flat across the $N$-photon threshold. This is illustrated in Fig. 5 for ionization of hydrogen over a range of laser-field intensities in the vicinity of the 14th photon channel closing. A very similar behavior of $s_{2}$ is observed at other channel closings for hydrogen, as well as for neon. Basically, $s_{2}$ decreases abruptly with increasing orbital angular momentum $l_{2}$ in the open channel, so that for large orbital angular momentum $l_{2}$ in the open channels, the Rydberg resonances should be suppressed. Since large

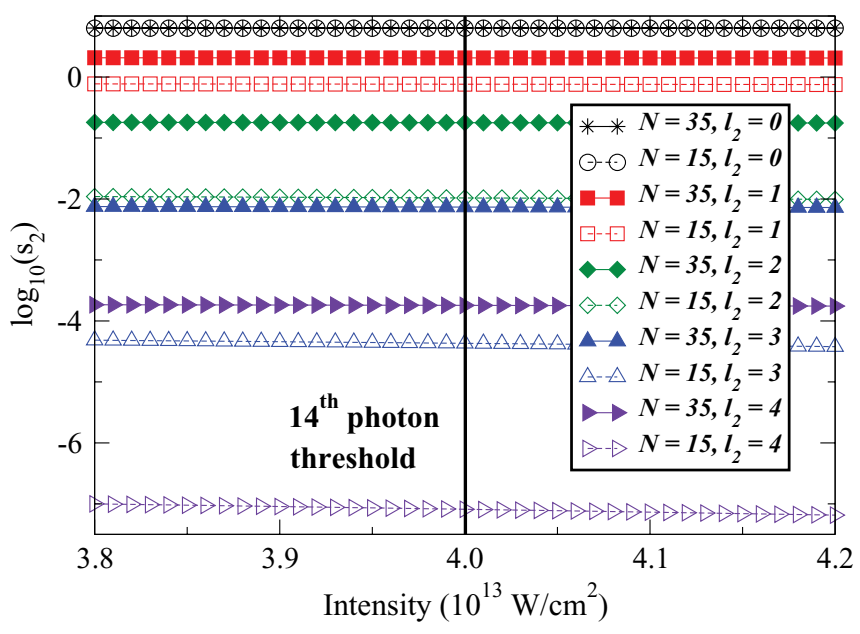

FIG. 5. (Color online) Behavior of $s_{2}$ [cf. Eq. (23)] in the $N=15$ and $N=35$ open channels for ionization of hydrogen by a $1 \mu \mathrm{m}$ linearly polarized laser field for a range of laser intensities below and above the 14th photon threshold (denoted by the vertical solid line). Each nearly horizontal line corresponds to the value of $s_{2}$ for a particular value of the orbital angular momentum $l_{2}$ in either the $N=15$ or the $N=35$ channel, as specified in the legend. Note that the two lines for $l_{2}=0$ are nearly identical on the scale of the figure. 
values of $l_{2}$ in the open channels occur primarily by resonant transitions via intermediate Rydberg states of large angular momentum $l$ (because Rydberg states $n l$ with lower orbital angular momenta $l$ and large $n$ are rapidly ionized), this seems to be a contributing factor to the flat behavior of the ionization yield above the threshold.

With regard to such suppression of the Rydberg resonances, it was suggested in Refs. [23,32] that both the resonant excitation amplitudes for $1 s-n l$ transitions and the ionization amplitudes from $n l$ resonant states decrease rapidly with increasing principal quantum number $n$. A second possibility mentioned in [32] is that since the resonant intensity decreases with increasing $n$, a smaller ionization rate should be observed when going through resonances with higher $n$ Rydberg states. However, from Table I one observes that the laser-field intensity varies by only a tiny fraction over the region of the Rydberg resonances. It thus seems unlikely that such a small variation of the laser-field intensity causes the heights of the resonant peaks to vanish abruptly with increasing $n$. The first set of reasons thus appears more likely. To illustrate this, recall that in the case under consideration in Sec. V A2 the resonant spikes are due to 15 -photon resonant $1 s \rightarrow n l$ transitions. It is well known that the single-photon oscillator strengths for these transitions decrease with $n$ as $n^{-3}$ [39]. Thus one may expect to observe a similar power law for the ground-state multiphoton ionization rate at a resonance with the $n$th excited state. Our rough estimates show reasonably good agreement with the $n^{-3}$ law. In fact, the agreement is reasonably good for resonances above the odd-photon thresholds in the case of hydrogen. For resonances above the even-photon thresholds the agreement with the $n^{-3}$ law is worse. Thus, in addition to $n$ suppression, which seems to describe well the ionization spectra for weak laser fields (i.e., when the ac Stark shift is negligible [23]), for strong laser fields we also find evidence for $s_{2}$ suppression [i.e., suppression of the dominant resonant peaks corresponding to resonances with high $l$ Rydberg states (cf. also [31,32])]. In the case of neon, the relative agreement of the ionization rates with the $n^{-3}$ law above the even- and odd-photon thresholds is reversed from the tendency found for $\mathrm{H}$. In general, we find a distinct dependence of our results on the parity of the transition, which will be discussed later.

\section{B. Present analysis of an effective threshold}

Within the SAE approximation, the threefold pattern in the total ionization rate spectrum seems to be a general property of the spectrum of any atom (cf. the results in Sec. IV and in Refs. [31,32]). Its origin for ionization by intense laser fields has been explained in Sec. V A. We emphasize here the most intriguing feature of this pattern: that the resonance regions in the total ionization rate spectrum are shifted with respect to the corresponding thresholds. As explained in Sec. V A, this shift results from interaction of the laser field with high-energy Rydberg levels. We show below that over the range of laserfield intensities considered in Figs. 2-4, the extent of the shift for both $\mathrm{H}$ and $\mathrm{Ne}$ varies only very slowly with intensity.

A key feature of the ionization rate spectra shown in Figs. 2-4 (as well as in the theoretical and experimental results in Refs. [31,32]) is that the first resonance following each multiphoton threshold occurs quite far above the threshold, i.e., for a laser-field intensity significantly higher than the threshold intensity. One may thus conclude that the dressed Coulomb potential acts as if the ionization threshold is effectively shifted to lower energies so as to support only a finite number of bound states. For this reason the ionization spectra show only a finite number of Rydberg resonances.

Our calculations for ionization of $\mathrm{H}$ by a $1000 \mathrm{~nm}$ laser field show that the threshold energy shift is near resonance with the $n=9$ excited energy level, over the range of intensities considered in Figs. 2 and 3, which corresponds to a range of the Keldysh parameter, $\gamma=\sqrt{\left|E_{0}\right| / 2 U_{p}}$, that varies between 0.95 and 1.71 , which is in the multiphoton regime. We estimate that the energy shift varies between $13 \%$ and $14 \%$ of the photon energy. In the case of Ne ionized by an $800 \mathrm{~nm}$ laser field, the energy shift reaches resonance with the Rydberg state having the principal quantum number $n=12$. This gives an energy shift of approximately $6 \%-7 \%$ of the photon energy for the intensity range shown in Fig. 4. In this case, $1.01<\gamma<1.90$, which also corresponds to the multiphoton regime. Our results are confirmed by calculations of Shakeshaft and Potvliege [31,32] who considered ionization of $\mathrm{H}$. For a $616 \mathrm{~nm}$ laser field, the range of intensities considered in [31,32] was such that $1.26<\gamma<4.38$, i.e., in the multiphoton regime, and the effective threshold reached resonance with the $n=9$ Rydberg states. Results for a near-infrared $1064 \mathrm{~nm}$ laser field were also given in [31,32], with $1.47<\gamma<3.60$. Rydberg resonances with $n$ up to 8 were observed. This suggests a threshold energy shift of $\approx 0.008$ a.u.

\section{Prior analyses of an effective threshold}

Different arguments and interpretations are given in the literature to explain the threshold shift that is observed in both multiphoton ionization and high-harmonic generation processes. Those arguments, some of which are reviewed below, lead to various estimates for the energy shift. For convenience, we summarize some of these estimates in Table II.

Proposals for introducing an effective ionization potential when describing strong-field processes have been put forth previously in the context of explaining the resonantlike enhancements of strong-field ATI spectra that have been observed to occur when only small changes are made in

TABLE II. Estimates for the energy shift of the ionization threshold given in a number of references.

\begin{tabular}{lccccc}
\hline \hline Reference & Atom & $\begin{array}{c}E_{0} \\
\text { (a.u.) }\end{array}$ & $\begin{array}{c}\omega \\
\text { (a.u.) }\end{array}$ & $\begin{array}{c}\text { Keldysh } \\
\text { parameter }\end{array}$ & $\begin{array}{c}\text { Energy shift } \\
\text { (a.u.) }\end{array}$ \\
\hline$[2,3]$ & $\mathrm{Ar}$ & 0.579 & 0.057 & $0.82<\gamma<2.46$ & 0.04 \\
{$[4]$} & $\mathrm{Ne}$ & & 0.0006 & $0.86<\gamma<1.66$ & 0.015 \\
{$[24]$} & $\mathrm{Ne}$ & 0.792 & 0.0022 & $\gamma \sim 0.71$ & 0.042 \\
This work & $\mathrm{Ne}$ & & 0.057 & $1.01<\gamma<1.90$ & 0.004 \\
{$[15]$} & $\mathrm{H}$ & & 0.0456 & $0.40<\gamma<1.00$ & 0.114 \\
{$[16]$} & $\mathrm{H}$ & & 0.0234 & $0.035<\gamma<0.80$ & $0.007-0.015$ \\
{$[31,32]$} & $\mathrm{H}$ & 0.5 & 0.074 & $1.26<\gamma<4.38$ & 0.006 \\
{$[31,32]$} & $\mathrm{H}$ & & 0.043 & $1.47<\gamma<3.60$ & 0.008 \\
This work & $\mathrm{H}$ & & 0.0456 & $0.95<\gamma<1.71$ & 0.006 \\
\hline \hline
\end{tabular}


the laser-field intensity [2,17-21]. By analyzing threshold phenomena for the case of a finite-range potential, similar enhancement effects were obtained in ATD spectra $[2,3,5]$. Such approaches found enhancements also when studying the intensity and/or wavelength dependence of high-harmonic spectra at channel closings [2-4,15,46,47]. These successes in reproducing resonantlike enhancements in strong-field processes for the case of a short-range potential suggested that for neutral atoms, a reduced effective ionization potential $\tilde{E}_{0}$ should be introduced, instead of $E_{0}$, the basic idea being that in a strong laser field, high-lying energy levels are distorted such that they form an effective continuum that begins lower in energy as compared to the true continuum $[2-4,15]$.

A variety of ways to estimate the magnitude of the ionization threshold shift have been proposed. Some authors propose that one should examine the field-free Rydberg spectrum of an atom and, if there are gaps in the spectra, to choose the effective threshold as beginning at the energy of the onset of the gap between Rydberg levels that lies closest to the field-free threshold $[2,3]$. Others propose that the ionization potential shift should equal the energy $\tilde{E}_{0}$ of the Rydberg state whose radius is closest to the excursion amplitude $\alpha_{0}$ of the active electron in the laser field [4]. Still others propose to lower the ionization threshold to the energy at which the widths of neighboring Rydberg levels in the laser field become comparable to the distance between the field-free Rydberg state energies [15].

Even though the idea of an effective continuum threshold is appealing and physically understandable, in all of these studies the respective energy shift has been overestimated. For this reason, a different interpretation of this energy shift has been proposed in Ref. [16], in which it is related to the effect of the Coulomb potential on the motion of rescattered photoelectrons. The necessity of reducing the ionization potential in the case when the active electron in a strong laser field experiences an attractive Coulomb field has been noted by others (see, e.g., Refs. [23,24]), although not in the context of channel-closing effects on ATI spectra, which is the focus of the references we have cited above $[2-4,15,16]$.

In summary, there are ambiguities regarding both the physical origin of a shift of the ionization potential of an atom in an intense laser field and its magnitude. By analyzing the threshold behavior of ionization rates in the presence of a laser field, we have shown in Secs. V A3 and V B that the nearly flat part of the ionization spectrum originating from the interaction of the laser field with high-energy Rydberg states mimics an energy shift of the ionization threshold. Furthermore, the magnitude of this shift has only a weak dependence on the laser-field intensity, in agreement with the prediction of Ref. [16].

Based on the analysis of the threshold behavior of ATI spectra in the multiphoton regime presented in Sec. III, none of the other explanations appear to be valid (at least in the multiphoton regime) since they assume that high Rydberg states are rapidly ionized. The dominant interaction controlling the threshold behavior is the diagonal Coulomb potential, since the electromagnetic coupling does not affect the threshold features, particularly the near-threshold Rydberg series in the ionization rates [23]. The physics behind this is clear: An electron in a highly excited Rydberg state with an orbital period that is large compared to the period of the laser field, is located predominantly at large distances from the nucleus, where the electromagnetic coupling is weak. This contrasts with the case of a Rydberg electron in a static electric field, whereby the ionization rate grows rapidly with $n$. In a laser field, however, the electromagnetic coupling to highly excited Rydberg states drops very fast with $n$. Moreover, the amplitude of oscillations stemming from a Rydberg series also decreases rapidly with $n$, and becomes unnoticeable above a certain value of $n$. In particular, Gratl et al. [23] observed a rapid decrease in the oscillation amplitude of Rydberg levels for laser fields in which the ac Stark shifts were negligible. They also found that these oscillations can be suppressed due to the finite duration of a laser pulse. In the present investigation the laser pulse is infinitely long, so that the only reason for such oscillation suppression is the decreasing electromagnetic coupling to higher Rydberg states (and, specifically, to those Rydberg states with high orbital angular momenta).

\section{Parity effects on the spectra}

As noted above, the ionization rates show a very pronounced parity dependence. This is particularly noticeable in the spectrum of hydrogen (cf. Fig. 3), involving the ionization of an $s$-valence electron. In this case, the resonant structures in region " $\mathrm{B}$ " that follow the closing of an odd-photon threshold [cf. Figs. 3(a) and 3(c)] involve quite broad peaks, in contrast to the resonant structures that follow the closing of even-photon thresholds [cf. Figs. 3(b) and 3(d)], which involve quite narrow peaks. In the spectra of neon, involving the ionization of a $p$-valence electron, the situation is reversed (Fig. 4): Wider resonances are observed for laser intensities above the closing of even photon channels, and narrower resonances are observed above the closing of odd-photon resonances. These two cases are consistent with the following parity selection rule: The widths of resonances above the closing of an $N$-photon channel are broader if the resonance states have odd parity and they are narrower if they have even parity.

A second parity selection rule applies to region " $\mathrm{C}$ " of the ionization spectra: There is a sharp rise of the total ionization rate above the closing of an $\mathrm{N}$-photon threshold for laser intensities above the resonance region when the resonance states have odd parity, whereas there is no sharp rise when the resonance states have even parity. This second selection rule may be observed in the spectra of both the $\mathrm{H}$ atom and the $\mathrm{Ne}$ atom. For H, see. Figs. 3(a) and 3(c). For Ne, see the spectra beyond the resonance region above the closing of the $N=16$ and $N=18$ thresholds in Fig. 4.

The physical reasons for these two observed parity selection rules remain speculative. However, one can adduce some relevant physical facts. First, we note that the states in the lowest open channel above the closed $N$-photon channel [i.e., the states of the $(N+1)$-photon channel, which contributes most to the total ionization signal] are even-parity states for those $N$-photon channels having odd-parity resonances. These even-parity continuum states most likely overlap with the ionic atomic core more than the odd-parity continuum states. Perhaps this greater overlap results in wider intermediate $(N$-photon) state resonant structures. 
Second, we note that analyses of ionization rates near multiphoton thresholds in the case of negative atomic ions also found effects related to the parity of the active electron $[5-9,13]$. It was demonstrated that one can observe these parity effects in the enhanced ionization signal for both hydrogen $(s$-valence electron) and fluorine ( $p$-valence electron) negative ions $[5-9,13]$ when channels corresponding to the absorption of an even or an odd number of laser photons are closing, respectively. This has been interpreted as a manifestation of the Wigner threshold law [22] for ionization from short-range potentials. In what way these parity effects in the spectra of negative ion detachment processes may be related to the parity effects observed in this paper for ATI of neutral atoms, however, remains unclear.

\section{SUMMARY AND CONCLUSIONS}

In this paper we have presented a theory for the nearthreshold behavior of above-threshold ionization rates with an emphasis on the role of the laser field. This theory is illustrated by numerical results for ionization of hydrogen and neon atoms (in the multiphoton regime). Motivation for this work stems from recent widespread interest devoted to the threshold behavior of above-threshold ionization or high-harmonic generation processes, with a focus on a downward shift of the ionization potential (see, for instance, Refs. [3,4,15,16,24]).

We show in this paper that in a strong laser field the total ionization rates for neutral atoms exhibit a typical threefold pattern over the range of intensities whereby the ponderomotive shift changes by the energy of one photon. We interpret this pattern as due to the coupling of the laser field with atomic levels that have high, moderate, and low orbital angular momenta (going in the direction of increasing laser-field intensity). The most intriguing feature of the ionization rates is their nonoscillatory behavior above the threshold, which is consistent with the idea of an effectively lowered ionization continuum. We attribute this flattened region of the ATI spectrum (having the appearance of a lowered ionization potential) to the rapid decrease of the laser-field interaction with high $n$ Rydberg states, especially those having high orbital angular momenta. We find that this flattened spectrum (giving the appearance of an apparent shift of the threshold) depends on the laser field and is a universal feature observable in the ATI spectrum of any atomic target.

Beyond the flat region of the ATI spectrum above the closing of an $N$-photon threshold, we attribute the observed oscillatory structures to resonances with those excited Rydberg states that have moderate orbital angular momenta. These structures exhibit a clear dependence on the parity of the active electron: wide peaks for the odd-parity states in the closing $N$-photon channels, and narrow peaks for closing channels having evenparity states. This parity selection rule is a general feature of the oscillatory structures above the ionization thresholds for both atomic systems (i.e., $\mathrm{H}$ and $\mathrm{Ne}$ ) for which we have presented results, which have, respectively, $s$ - and $p$-valence electrons. A second feature of the ATI spectra also satisfies a parity selection rule for laser intensities corresponding to spectra above the resonance region. Namely, the total ionization rate exhibits a pronounced upward step for the odd-parity states in the closing channels, but not nearly so pronounced for the case of even-parity states.

Finally, we note that the results of this paper and in Refs. [31,32] show a profound agreement of the numerical multiphoton Sturmian-Floquet calculations and the analytical predictions presented here based on collision theory for the case of an attractive Coulomb potential. We have shown that both the apparent ionization potential shift and the structure of the observed Rydberg resonances are threshold-related effects, and that the laser-field coupling plays a crucial role in their formation. More detailed features of ionization rates such as the energy shifts of Rydberg resonances and selection rules for their dependence on the parity of the active electron have also been presented and discussed.

\section{ACKNOWLEDGMENTS}

This work is supported in part by the U.S. National Science Foundation under Grants No. PHY-0969381 (I.I.F.) and No. PHY-1208059 (A.F.S.). K.K. gratefully acknowledges the hospitality of the Department of Physics and Astronomy at the University of Nebraska, Lincoln, NE, USA.
[1] H. R. Sadeghpour, J. L. Bohn, M. J. Cavagnero, B. D. Esry, I. I. Fabrikant, J. H. Macek, and A. R. P. Rau, J. Phys. B 33, R93 (2000).

[2] G. G. Paulus, F. Grasbon, H. Walther, R. Kopold, and W. Becker, Phys. Rev. A 64, 021401(R) (2001).

[3] R. Kopold, W. Becker, M. Kleber, and G. G. Paulus, J. Phys. B 35, 217 (2002).

[4] C. Figueira de Morisson Faria, R. Kopold, W. Becker, and J. M. Rost, Phys. Rev. A 65, 023404 (2002).

[5] B. Borca, M. V. Frolov, N. L. Manakov, and A. F. Starace, Phys. Rev. Lett. 88, 193001 (2002).

[6] K. Krajewska, I. I. Fabrikant, and A. F. Starace, Bull. Am. Phys. Soc. 51, 119 (2006).

[7] N. L. Manakov and M. V. Frolov, Pis'ma Zh. Eksp. Teor. Fiz. 83, 630 (2006) [JETP Lett. 83, 536 (2006)].
[8] K. Krajewska, I. I. Fabrikant, and A. F. Starace, Phys. Rev. A 74, 053407 (2006). Note that there are misprints in Eq. (32) of Ref. [8]. The correct form of this equation is

$$
T_{i}=\sqrt{s_{i}} \frac{\left(M_{j j}-i s_{j}\right) g_{i}-M_{12} g_{j}}{\operatorname{det} M-i s_{i} M_{j j}-i s_{j} M_{i i}-s_{1} s_{2}},
$$

which fully agrees with Eq. (22) of the present paper if we put $C_{i, l_{i}}=1$ for $i=1,2$. Also in Ref. [8], the definition of the coefficient $c_{1}$ in Eq. (34) should be changed to $c_{1}=\left(M_{22} g_{1}-\right.$ $\left.M_{12} g_{2}\right)^{2}+\left(s_{2} g_{1}\right)^{2}$, whereas Eq. (36) should read

$$
\left|T_{2}^{2}\right|=s_{2}\left(\frac{c_{2}+\alpha \kappa}{a+\beta \kappa}+O\left(\kappa^{2}\right)\right) .
$$

We stress that these misprints do not affect the discussion in Ref. [8] following the corrected equations. 
[9] K. Krajewska, I. I. Fabrikant, and A. F. Starace, Laser Phys. 17, 368 (2007).

[10] D. B. Milošević, E. Hasovič, M. Busuladžić, A. GazibegovićBusuladžić, and W. Becker, Phys. Rev. A 76, 053410 (2007).

[11] E. Hasovič, M. Busuladžić, A. Gazibegović-Busuladžić, D. B. Milošević, and W. Becker, Laser Phys. 17, 376 (2007).

[12] D. B. Milošević, E. Hasovič, S. Odžak, M. Busuladžić, A. Gazibegović-Busuladžić, and W. Becker, J. Mod. Opt. 55, 2653 (2008).

[13] K. Krajewska, I. I. Fabrikant, and A. F. Starace, Phys. Rev. A 78, 023407 (2008).

[14] H. G. Muller, Opt. Express 8, 44 (2001).

[15] M. V. Frolov, N. L. Manakov, and A. F. Starace, Phys. Rev. Lett. 100, 173001 (2008).

[16] K. L. Ishikawa, K. Schiessl, E. Persson, and J. Burgdörfer, Phys. Rev. A 79, 033411 (2009).

[17] P. Hansch, M. A. Walker, and L. D. Van Woerkom, Phys. Rev. A 55, R2535 (1997).

[18] M. P. Hertlein, P. H. Bucksbaum, and H. G. Muller, J. Phys. B 30, L197 (1997).

[19] M. J. Nandor, M. A. Walker, L. D. Van Woerkom, and H. G. Muller, Phys. Rev. A 60, R1771 (1999).

[20] E. Cormier, D. Garzella, P. Breger, P. Agostini, G. Chériaux, and C. Leblanc, J. Phys. B 34, L9 (2000).

[21] F. Grasbon, G. G. Paulus, H. Walther, P. Villoresi, G. Sansone, S. Stagira, M. Nisoli, and S. De Silvestri, Phys. Rev. Lett. 91, 173003 (2003).

[22] E. P. Wigner, Phys. Rev. 73, 1002 (1948).

[23] H. Gratl, G. Alber, and P. Zoller, J. Phys. B 22, L547 (1989).

[24] M. Yu. Ivanov, T. Brabec, and N. Burnett, Phys. Rev. A 54, 742 (1996).

[25] R. M. Potvliege and R. Shakeshaft, in Atoms in Intense Laser Fields, edited by M. Gavrila (Academic Press, Boston, 1992), p. 373.
[26] R. Shakeshaft and X. Tang, Phys. Rev. A 36, 3193 (1987).

[27] R. M. Potvliege and R. Shakeshaft, Phys. Rev. A 38, 1098(R) (1988).

[28] R. M. Potvliege and R. Shakeshaft, Phys. Rev. A 38, 4597 (1988).

[29] R. M. Potvliege and R. Shakeshaft, Phys. Rev. A 38, 6190 (1988).

[30] R. M. Potvliege and R. Shakeshaft, Phys. Rev. A 40, 3061 (1989).

[31] M. Dörr, R. M. Potvliege, and R. Shakeshaft, J. Opt. Soc. Am. B 7, 433 (1990).

[32] H. Rottke, B. Wolff-Rottke, D. Feldmann, K. H. Welge, M. Dörr, R. M. Potvliege, and R. Shakeshaft, Phys. Rev. A 49, 4837 (1994).

[33] R. M. Potvliege, Comput. Phys. Commun. 114, 42 (1998).

[34] A. M. Lane and R. G. Thomas, Rev. Mod. Phys. 30, 257 (1958).

[35] A. I. Baz', Ya. B. Zel'dovich, and A. M. Perelomov, Scattering, Reactions and Decays in Nonrelativistic Quantum Mechanics (Israel Program for Scientific Translation, Jerusalem, 1966).

[36] M. H. Ross and G. L. Shaw, Ann. Phys. 13, 147 (1961).

[37] M. Gailitis, Sov. Phys. JETP 17, 1328 (1963).

[38] A. I. Baz', Sov. Phys. JETP 6, 709 (1958).

[39] A. Giusti-Suzor and P. Zoller, Phys. Rev. A 36, 5178 (1987).

[40] P. G. Burke, P. Francken, and C. J. Joachain, J. Phys. B 24, 761 (1991).

[41] M. J. Seaton, Rep. Prog. Phys. 46, 167 (1983).

[42] C. Greene and Ch. Jungen, Adv. At. Mol. Phys. 21, 51 (1985).

[43] H. G. Muller and F. C. Kooiman, Phys. Rev. Lett. 81, 1207 (1998).

[44] H. G. Muller, Phys. Rev. A 60, 1341 (1999).

[45] X. M. Tong and C. D. Lin, J. Phys. B 38, 2593 (2005).

[46] B. Borca, A. F. Starace, A. V. Flegel, M. V. Frolov, and N. L. Manakov, Phys. Rev. A 65, 051402(R) (2002).

[47] D. B. Milošević and W. Becker, Phys. Rev. A 66, 063417 (2002). 